\title{
Characterization of an Adaptive Refinement Algorithm for a Meshless Eigenvalue Solver Based on Radial Basis Functions
}

\author{
Thomas Kaufmann*, Christian Engström*, Christophe Fumeaux ${ }^{\dagger}$ \\ *Laboratory for Electromagnetic Fields and Microwave Electronics (IFH), ETH Zurich, \\ Gloriastrasse 35, 8092 Zurich, Switzerland \\ ${ }^{\dagger}$ School of Electrical and Electronic Engineering, The University of Adelaide, SA 5005, Australia \\ Corresponding Email: thomas.kaufmann@ifh.ee.ethz.ch
}

\begin{abstract}
A meshless method based on a radial basis collocation approach is presented to calculate eigenvalues for the secondorder wave equation. Instead of an explicit mesh topology only a node distribution is required to calculate electric fields, thus facilitating dynamic alteration of the discretization of an electromagnetic problem. An algorithm is presented that automatically adapts an initially very coarse discretization by adding points where higher accuracy is required by the physics of the problem. The algorithm is applied to a cylindrical cavity resonator and the rate of convergence is compared to uniform refinements with the radial basis method and to a regular grid-based finite-difference approach.
\end{abstract}

\section{INTRODUCTION}

In computational electromagnetics, commonly used domaindiscretization solvers are based on finite-differences in timedomain (FDTD), or the finite elements method (FEM) in frequency-domain. FDTD is a second-order algorithm, i.e. the errors decrease at most with the the square of the grid spacing. FEM is usually used with low-order basis functions of polynomial degree 1 or 2 . The simulation error for a structured mesh is then comparable to FDTD for similar discretizations. Meshless methods based on a radial basis collocation approach are an emerging class of numerical schemes with advantages in accuracy over low order methods such as FEM/FDTD, but sharing the ease of implementation and flexibility of these methods. No explicit mesh configuration is required to solve physical problems with a meshless method. Instead, a set of nodes is used in a collocation approach, thus avoiding the computational overhead of dealing with mesh elements. This advantage is especially attractive for adaptive node refinement. Due to the unstructured nature of the interpolation algorithm, nodes can be placed in a arbitrary manner, i.e. complex and multi-scale geometries can be modeled accurately by an appropriate node distribution.

The approach with radial basis functions (RBFs) is sometimes referred to as the Radial Point Interpolation Method (RPIM) [1] or the Kansa method [2]. The collocation approach leads to a true meshless method where no volume integration is required, in contrast to a Galerkin approach [3]. Here, only the position of the collocation nodes is required. It has been estimated [4], that this type of methods can offer convergence at much higher rates than FDTD or low-order finite elements (FEM). This means that, in order to solve problems in electromagnetics, much coarser discretizations can be used to achieve the required accuracy, which results either in a shorter computation time or in an increase of the accuracy of a simulation at the same cost.

The goal of our research is to develop a versatile fullwave solver for 3D problems. Steps in that direction were the presentation of a RBF collocation time-domain method in two dimensions [5], as well as the development of an alternate direction implicit (ADI) approach for overcoming the CFL limit [6] and an implementation in three dimensions [7] by Yu and Chen.

This publication is structured as follows. Sec. II summarizes the interpolation method with radial basis functions. The eigenvalue solver for the Helmholtz equation is presented in Sec. III. In the subsequent Sec. IV, the adaptation algorithm is introduced. A numerical experiment illustrates the abilities of the algorithm in Sec. V and the paper is concluded in Sec. VI.

\section{RADIAL BASIS FUNCTIONS}

RBFs are an elaborate type of basis functions for highly accurate interpolation of scattered data. This kind of basis function is becoming more popular in many fields of science such as statistics and neural networks. They are also an interesting choice of basis functions to solve partial differential equations. The interpolation scheme is explained in detail in [1]. Hence, only a short summary is given here. A field component $u(\mathbf{x})$ at position $\mathbf{x}$ in a computational domain containing $N$ nodes is interpolated as

$$
\langle u(\mathbf{x})\rangle=\sum_{n=1}^{N} a_{n} r_{n}(\mathbf{x})=\mathbf{r}(\mathbf{x})^{T} \mathbf{a},
$$

with the radial basis functions

$$
r_{n}(\mathbf{x})=\exp \left(-\alpha_{c} \frac{\left|\mathbf{x}_{n}-\mathbf{x}\right|^{2}}{d_{c}^{2}}\right)
$$

of Gaussian type. The shape parameter $\alpha_{c}$ is used to control the flatness of the basis function (Fig. 1). The normalization 


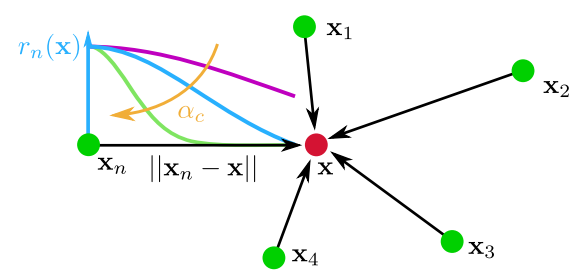

Fig. 1: Gaussian radial basis functions are used to interpolate the field value at $\mathrm{x}$, based on the field values at the surrounding node locations $\mathbf{x}_{n}$. The shape parameter $\alpha_{c}$ is used to control the flatness of the basis functions.

factor $d_{c}$ denotes the average node distance inside the computational domain. Here, we interpolate values in 2D with $\mathbf{x}=(x, y)$.

The interpolation parameter vector $\mathbf{a}$ is calculated in a preprocessing step as follows. A matrix is set up to interpolate the field values in the nodes $\mathbf{U}_{s}=\left[u\left(\mathbf{x}_{1}\right), u\left(\mathbf{x}_{2}\right), \ldots, u\left(\mathbf{x}_{N}\right)\right]^{T}$. This system can be written in the form

$$
\left(\begin{array}{c}
u\left(\mathbf{x}_{1}\right) \\
\vdots \\
u\left(\mathbf{x}_{N}\right)
\end{array}\right)=\left(\begin{array}{ccc}
r_{1}\left(\mathbf{x}_{1}\right) & \cdots & r_{1}\left(\mathbf{x}_{N}\right) \\
\vdots & \ddots & \vdots \\
r_{N}\left(\mathbf{x}_{1}\right) & \cdots & r_{N}\left(\mathbf{x}_{N}\right)
\end{array}\right)\left(\begin{array}{c}
a_{1} \\
\vdots \\
a_{N}
\end{array}\right)
$$

which we denote in compact notation as

$$
\mathbf{U}_{s}=\mathbf{R}_{0} \mathbf{a} \text {. }
$$

When the interpolation matrix $\mathbf{R}_{0}$ is non-singular, the interpolation parameter a can be calculated as

$$
\mathbf{a}=\mathbf{R}_{0}^{-1} \mathbf{U}_{s} \text {. }
$$

$\mathbf{R}_{0}$ can become singular when two nodes coincide, or when the basis functions are too flat, leading to a unitary matrix. The shape functions $\Psi(\mathbf{x})$ and their derivatives are subsequently obtained as

$$
\begin{aligned}
\langle u(\mathbf{x})\rangle & =\mathbf{r}^{T}(\mathbf{x}) \mathbf{a}=\mathbf{r}^{T}(\mathbf{x}) \mathbf{R}_{0}^{-1} \mathbf{U}_{s} \\
& =\mathbf{\Psi}(\mathbf{x}) \mathbf{U}_{s} \\
& =\sum_{j=1}^{N} \Psi^{j}(\mathbf{x}) u\left(\mathbf{x}_{j}\right), \\
\left\langle\partial_{\kappa} u(\mathbf{x})\right\rangle & =\partial_{\kappa} \mathbf{r}^{T}(\mathbf{x}) \mathbf{a}=\partial_{\kappa} \mathbf{r}^{T}(\mathbf{x}) \mathbf{R}_{0}^{-1} \mathbf{U}_{s} \\
& =\partial_{\kappa} \Psi(\mathbf{x}) \mathbf{U}_{s} \\
& =\sum_{j=1}^{N} \partial_{\kappa} \Psi^{j}(\mathbf{x}) u\left(\mathbf{x}_{j}\right), \\
\left\langle\partial_{\kappa}^{2} u(\mathbf{x})\right\rangle & =\partial_{\kappa}^{2} \mathbf{r}^{T}(\mathbf{x}) \mathbf{a}=\partial_{\kappa}^{2} \mathbf{r}^{T}(\mathbf{x}) \mathbf{R}_{0}^{-1} \mathbf{U}_{s} \\
& =\partial_{\kappa}^{2} \Psi(\mathbf{x}) \mathbf{U}_{s} \\
& =\sum_{j=1}^{N} \partial_{\kappa}^{2} \Psi^{j}(\mathbf{x}) u\left(\mathbf{x}_{j}\right)
\end{aligned}
$$

with $\partial_{\kappa}$ the spatial derivative in $\kappa=x, y$ direction. The shape function $\Psi(\mathbf{x})=\left[\Psi^{1}(\mathbf{x}), \ldots, \Psi^{N}(\mathbf{x})\right]$ fulfills the delta

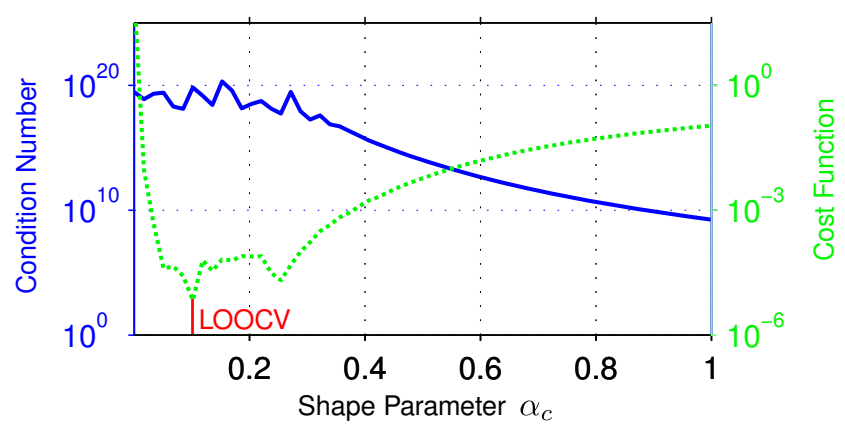

Fig. 2: Relation between condition number of interpolation matrix and the cost error function to be minimized in LOOCV algorithm for different shape parameters $\alpha_{c}$.

property

$$
\Psi^{j}\left(\mathbf{x}_{i}\right)=\left\{\begin{array}{ll}
0, & i \neq j \\
1, & i=j
\end{array},\right.
$$

i.e. the values at the nodes are fitted exactly.

The choice of the shape parameter $\alpha_{c}$ is a crucial step in order to achieve accurate results. Large values of $\alpha_{c}$ lead to low accuracy. Increasingly small values of $\alpha_{c}$ on the other hand lead to flatter basis functions and increased accuracy [8], but also to an increasingly poorly conditioned interpolation matrix $\mathbf{R}_{0}$ [9]. At one point, $\mathbf{R}_{0}$ becomes ill-conditioned, leading to a failure of the numerical inversion for the interpolation matrix. This results in degenerate interpolation problems for the shape functions. Here, the "leave-one-out-cross-validation" algorithm (LOOCV) [10] is applied to find an optimized shape parameter for a given node distribution, i.e. the value of the shape parameter $\alpha_{c}$ where the numerical inversion leads to a maximized interpolation accuracy. The cost function $C F$ of this optimization is based on the pseudo-inverse [11] $\operatorname{pinv}(\cdot)$ of the interpolation matrix:

$$
\begin{aligned}
C F\left(\alpha_{c}\right) & =\max _{k}\left|e_{k}\left(\alpha_{c}\right)\right| \\
e_{k}\left(\alpha_{c}\right) & =\frac{\sum_{j=1}^{N} \operatorname{pinv}\left(\mathbf{R}_{0}\right)_{k, j} f^{t}\left(\mathbf{x}_{j}\right)}{\operatorname{pinv}\left(\mathbf{R}_{0}\right)_{k, k}}
\end{aligned}
$$

where $\mathbf{R}_{0}$ depends on the global shape parameter $\alpha_{c}$. The test function $f^{t}(\mathbf{x})$ is a smooth function, defined over the whole computational domain. In this case, we chose the twodimensional sinc-function

$$
f^{t}(x, y)=\operatorname{sinc}(x, y)=\left\{\begin{array}{cc}
\frac{\sin (\pi x)}{\pi x} \frac{\sin (\pi y)}{\pi y}, & x, y>0 \\
1, & x, y=0
\end{array} .\right.
$$

The optimization algorithm is used to find the minimum of the cost function $C F\left(\alpha_{c}\right)$. The relation between the condition number and the cost error function for an exemplary node distribution is shown in Fig. 2. In this case, the algorithm yields a value for the shape parameter of $\alpha_{c}=0.1$.

\section{EigenVAlue Solver}

In [12], an eigenvalue solver for the Helmholtz problem, based on compactly supported basis functions is presented. 
There the eigenmodes are solved for the interpolation coefficient vector a (1). A different approach is taken here, where we compute the shape functions $\boldsymbol{\Psi}(\mathbf{x})$ in a preprocessing step and solve for the eigenmodes, i.e. the resonance frequency and field distribution of the electric field at these frequencies. We consider a 2D transverse magnetic (TM) case, where the electric field points perpendicular to the computational domain $E_{z}(x, y)$. The discretized eigenvalue problem can be expressed as:

$$
\begin{aligned}
\mathcal{L}^{h} \mathbf{E}_{z} & =\lambda \mathbf{E}_{z} & & \text { in } \Omega \\
\mathbf{E}_{z} & =0 & & \text { on } \partial \Omega .
\end{aligned}
$$

with the wave equation inside the domain and a Dirichlet condition for perfect electric conductors (PEC) on the boundary. $\mathbf{E}_{z}$ is a vector with a length equal to the number of nodes and $\lambda=\omega^{2} / c_{0}^{2}$ are the eigenvalues. $\mathcal{L}^{h}$ contains the Laplace operator, discretized with the RBF interpolation shape functions $(6 \mathrm{c})$ :

$$
\mathcal{L}_{j, i}^{h}=\partial_{x}^{2} \Psi^{j}\left(\mathbf{x}_{i}\right)+\partial_{y}^{2} \Psi^{j}\left(\mathbf{x}_{i}\right) .
$$

The eigenproblem is solved with the numerical full matrix library ARPACK [13], and yields the eigenpairs $\left\{\mathbf{E}_{z}^{i}, \lambda^{i}\right\}$ with $i=1, \ldots, N$.

\section{AdAptive Algorithm}

The refinement algorithm in [14] for frequency-domain source problems is adapted to the present eigenvalue problem. A residual error is calculated on a set of test nodes $\mathbf{y}_{j}$ (Fig. 3), generated though a Delaunay tessellation of the initial set of collocation nodes. The test nodes are placed on the edge centers of the Delaunay cells, in between two existing collocation nodes. The residual error function is defined as

$\eta\left(\mathbf{y}_{j}\right)= \begin{cases}\nu_{1} d_{\mathbf{y}_{j}}^{2}\left|\lambda^{i} \mathbf{E}_{z}^{i}\left(\mathbf{y}_{j}\right)-\Delta \mathbf{E}_{z}^{i}\left(\mathbf{y}_{j}\right)\right| & \text { (internal node) } \\ +\nu_{2} d_{\mathbf{y}_{j}}^{2}\left|\mathbf{E}_{z}^{i}\left(\mathbf{y}_{j}\right)\right| & \text { (boundary node) }\end{cases}$

for a predefined eigenpair $\left\{\mathbf{E}_{z}^{i}, \lambda^{i}\right\}$. The weighting factors $\nu_{1}, \nu_{2}$ are used to set the importance of internal and boundary nodes. Numerical experiments showed that $\nu_{1}=1, \nu_{2}=1000$ leads to a good balance between the internal and boundary nodes. The residual error diminishes for very dense node distributions where the local Delaunay edge length $d_{\mathbf{y}_{j}}$ becomes very small.

Test node locations $\mathbf{y}_{j}$, where the residual error is larger than a predefined threshold

$$
\eta\left(\mathbf{y}_{i}\right) \geq \beta \max _{n} \eta\left(\mathbf{y}_{n}\right), \beta \in(0,1)
$$

are subsequently added to the set of collocation nodes. A value of $\beta=0.2$ proved to be a good value, i.e. all nodes with an error larger than $20 \%$ of the maximum error are added to the original set. This value is a trade-off between making the simulation less accurate by adding unnecessary nodes, and adding too few nodes, making it impossible to properly refine certain regions of the computational domain. In the next iteration step, the LOOCV algorithm is applied on the new collocation nodes to obtain an optimized shape parameter $\alpha_{c}$,



Fig. 3: Generation of set of test nodes $\mathbf{y}_{j}$, based on a Delaunay Tessellation of collocation nodes $\mathbf{x}_{i}$.

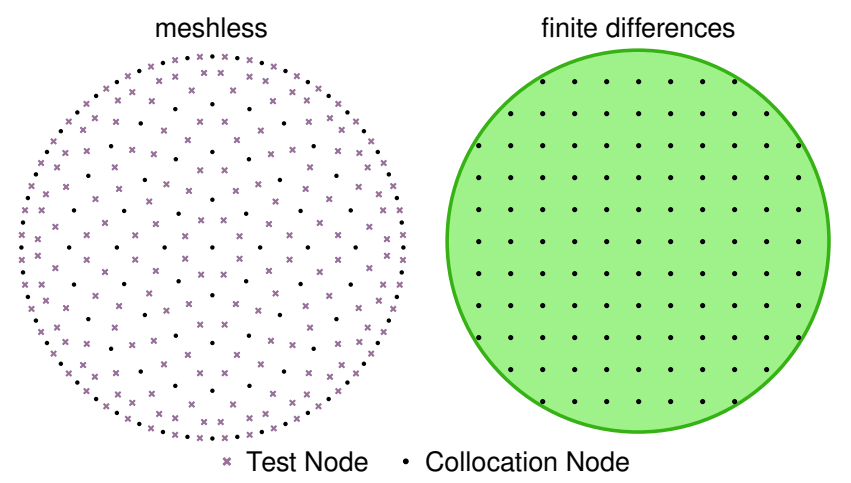

Fig. 4: Initial node distribution before node refinement for the meshless method (left) and the finite-difference method (right). The test nodes (x) are shown for the meshless method.

and the eigenvalue problem is solved again. This procedure is repeated until a required accuracy is achieved.

\section{NUMERICAL EXPERIMENT}

We calculate the resonance frequencies and eigenmodes of a cylindrical unit disc with perfectly conducting boundary conditions. The convergence rate is investigated. The RBF methods is initiated with a radially symmetric node distribution (Fig. 4). The proposed refinement algorithm is compared with uniform refinement, where in each step, the node distance is uniformly reduced, thus increasing the number of degrees of freedom. These approaches are compared to a uniformly refined secondorder finite-difference method on a regular rectangular grid.

Fig. 5 shows the node distribution after six iterations for a $T M_{02}$ mode. The adaptive algorithm adds only nodes in the locations where necessary. Remarkably in the region near boundaries, there is a significantly higher node density than in other regions. This conforms well with the findings of [15], where it was numerically shown that higher accuracy is achieved when additional nodes are placed near the boundary.

The accuracy is studied for the relative eigenvalue error

$$
\mathcal{E}_{\text {rel }}=\frac{\left|\lambda^{i}-\omega_{t h}^{2} / c_{0}^{2}\right|}{\omega_{t h}^{2} / c_{0}^{2}}
$$

where $\lambda_{i}$ is the obtained numerical eigenvalue, and $\omega_{t h}$ is the theoretical analytical resonance frequency calculated as [16]

$$
\omega_{t h}=p_{n m}\left(\frac{c_{0}}{\rho}\right)
$$




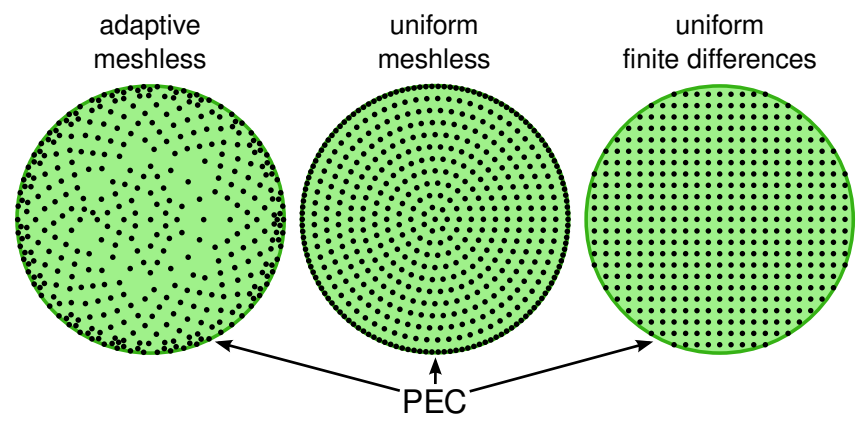

Fig. 5: Comparison between node distributions using adaptive (left) and uniform (center) refinement for the transverse magnetic mode $T M_{02}$. The rectangular finite difference grid is on the right.

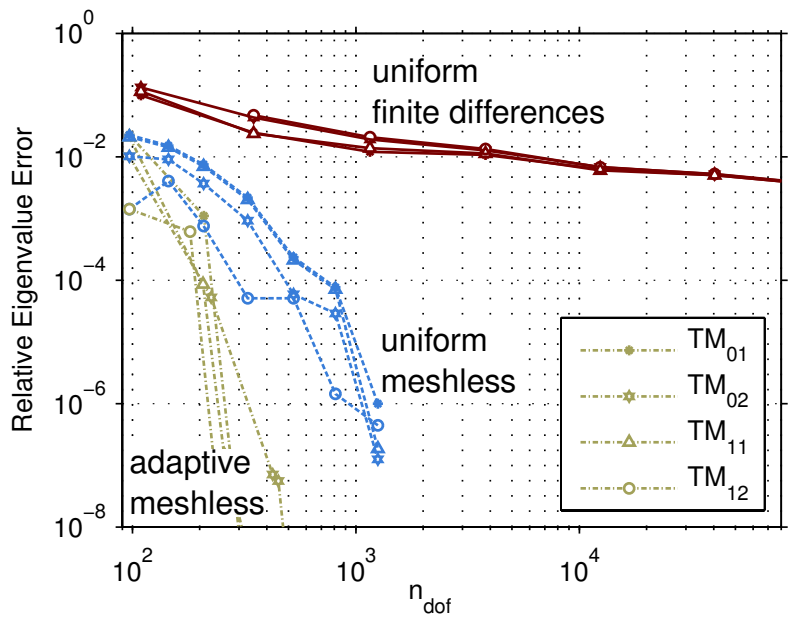

Fig. 6: Convergence of the relative eigenvalue error $\mathcal{E}_{\text {rel }}$ as a function of the number of degrees of freedom. A comparison between adaptive and uniform refinement for meshless and finite difference methods shows the much higher convergence rate for the proposed adaptive algorithm. Due to staircasing effects and low-order basis functions, the performance of the finite difference approach is inferior.

with $p_{m n}$ the $n t h$ zero of the $m t h$ order Bessel function $J_{m}\left(p_{m n}\right)=0, c_{0}$ the speed of light and $\rho$ the radius of the disc. Fig. 6 shows that the relative eigenvalue errors $\mathcal{E}_{\text {rel }}$ decrease rapidly when the number of nodes is increased through the proposed adaptive algorithm. A gain of five orders of magnitudes in accuracy is achieved when only tripling the number of nodes. The simulation with uniform refinement for the RBF method results in a slower rate of convergence, since nodes are added at locations where they are not necessary. The finite difference approach has errors much higher and slower convergence when comparing the number of degrees of freedom, i.e. the number of nodes. This is due to staircasing effects on the boundaries and the approximation with linear basis functions.

\section{CONCLUSION}

A meshless eigenvalue solver with adaptive refinement for a radial basis functions collocation method has been presented. In combination with an optimized shape parameter, this method yields excellent accuracy, superior to the commonly used finite-differences or low-order finite element methods. For quite coarse discretizations, highly precise simulation results can be achieved. The node refinement algorithm automatically places collocation nodes at locations where the contribution is relevant. Thus, a node distribution refined with the presented method increases the convergence rate. This refinement algorithm could be expanded using other error indicators, such as the information of the gradients, to further increase the efficiency of the adaptive refinement.

Meshless methods are a promising approach for solving electromagnetic problems due to the increased accuracy, ability to modeling complex geometries and the ease of handling a simple node distribution.

\section{REFERENCES}

[1] G. Liu and Y. Gu, An Introduction to Meshfree Methods and their Programming. Dortrecht, The Netherlands: Springer, 2005.

[2] E. Kansa, "Multiquadrics-a scattered data approximation scheme with applications to computational fluid-dynamics-II solutions to parabolic hyperbolic and elliptic partial differential equations," Computers \& Mathematics with Applications, vol. 19, no. 8-9, pp. 147-161, 1990.

[3] S. Beissel and T. Belytschko, "Nodal integration of the element-free Galerkin method," Computer Methods in Applied Mechanics and Engineering, vol. 139, no. 1-4, pp. 49-74, 1996.

[4] A. H.-D. Cheng, M. A. Golberg, E. J. Kansa, and G. Zammito, "Exponential convergence and $h-c$ multiquadric collocation method for partial differential equations," Numerical Methods for Partial Differential Equations, vol. 19, no. 5, pp. 571-594, 2003.

[5] T. Kaufmann, C. Fumeaux, and R. Vahldieck, "The meshless radial point interpolation method for time-domain electromagnetics," in IEEE MTTS Int. Microwave Symposium. Atlanta, GA, USA: IEEE, June 2008, pp. 61-65.

[6] Y. Yu and Z. D. Chen, "Towards the development of an unconditionally stable time-domain meshless method," Microwave Theory and Techniques, IEEE Transactions on, vol. 58, no. 3, pp. 578 -586, March 2010.

[7] _ _ "A 3-d radial point interpolation method for meshless time-domain modeling," IEEE Transactions on Microwave Theory and Techniques, vol. 57, no. 8, pp. 2015-2020, August 2009.

[8] T. Kaufmann, C. Fumeaux, and R. Vahldieck, "Parameter selection for the radial point interpolation time-domain method," in 25th International Review of Progress in Applied Computational Electromagnetics (ACES 2009), Monterey, USA, March 2009, pp. 395-400.

[9] M. D. Buhmann, Radial Basis Functions. New York, NY, USA Cambridge University Press, 2003.

[10] G. F. Fasshauer, Meshfree Approximation Methods with MATLAB. River Edge, NJ, USA: World Scientific Publishing Co., Inc., 2007.

[11] M. A. Rakha, "On the Moore-Penrose generalized inverse matrix," Applied Mathematics and Computation, vol. 158, no. 1, pp. 185 - 200, 2004.

[12] P. Kowalczyk and M. Mrozowski, "Mesh-free approach to Helmholtz equation based on radial basis functions," Journal of Telecommunications and Information Technology, 2005.

[13] R. Lehoucq, D. Sorensen, and C. Yang, ARPACK Users' Guide: Solution of Large-Scale Eigenvalue Problems With Implicityly Restarted Arnoldi Methods. Philadelphia, PA, USA: Society for Industrial and Applied Mathematics, 1998.

[14] B. B. Kee, G. Liu, G. Zhang, and C. Lu, "A residual based error estimator using radial basis functions," Finite Elements in Analysis and Design, vol. 44, no. 9-10, pp. 631-645, 2008.

[15] B. Fornberg, T. A. Driscoll, G. Wright, and R. Charles, "Observations on the behavior of radial basis function approximations near boundaries," Computers and Mathematics with Applications, vol. 43, no. 3, pp. 473490, February 2002.

[16] C. A. Balanis, Advanced Engineering Electromagnetics. New York, USA: John Wiley \& Sons, 1989. 